\title{
Timing of the Binary Pulsar B1259-63
}

\author{
N. Wang, ${ }^{1,2}$ S. Johnston ${ }^{1}$ and R. N. Manchester ${ }^{2}$ \\ ${ }^{1}$ School of Physics, University of Sydney, NSW 2006, Australia \\ ${ }^{2}$ Australia Telescope National Facility, CSIRO, NSW 1710, Australia
}

\begin{abstract}
This paper summarizes the results of 13 years of timing observations of a unique binary pulsar, B1259-63, which has a massive B2e star companion. A small glitch in the pulsar period apparently occurred in $1997 \mathrm{Aug}$, not long after the 1997 periastron. We found that spin-orbit coupling with secular changes in periastron longitude and projected semimajor axis cannot account for the observed period variations. A model in which step changes in pulsar orbital parameters occur at each periastron accounts best for the observed timing behavior.
\end{abstract}

\section{Introduction}

PSR B1259-63 was discovered in 1989 in a large-scale high frequency survey of the Galactic plane (Johnston et al. 1992a). It is the only known radio pulsar in orbit about a massive, main-sequence, B2e star (Johnston et al. 1992b). It has an orbital period of $\sim 1237$ days, and an eccentricity of 0.87 . The companion has a hot, tenuous polar wind and a cooler, high density, equatorial disk. The disk is likely to be highly tilted with respect to the pulsar orbital plane, and PSR B1259-63 is eclipsed for about 40 days as it goes behind the disk.

\section{Timing Results}

More than 1000 independent observations of PSR B1259-63 have been made by the Parkes 64-m radio telescope between 1990 Jan and 2003 Jun. The majority of the observations were made at frequencies around $1.4 \mathrm{GHz}$ with additional observations at $0.43,0.66,4.8,8.4$ and $13.6 \mathrm{GHz}$ (Johnston et al. 1996, 2001). This allows us to correct for dispersion measure variations occurring near periastra in the timing analysis. A glitch in the pulsar period apparently occurred near MJD 50691, about 94 days after the 1997 periastron. The total frequency change is $\Delta \nu_{g}=(67 \pm 1) \times 10^{-9} \mathrm{~s}^{-1}$ of which one third decayed exponentially with an assumed timescale of 100 days.

We initially fitted the entire data set using the five Keplerian orbital parameters and the spin frequency $(\nu)$ and its first two time-derivatives $(\dot{\nu}$ and $\ddot{\nu})$. The post-fit residuals shown in Figure 1(a) still have large systematic variations. We then added $\dot{\omega}$ and $\dot{x}$, where $\omega$ is the longitude of periastron and $x$ is the projected pulsar semi-major axis, to account for spin-orbit coupling as discussed by Wex et al. (1998). This model cannot account for the observations after the 1997 periastron (Fig. 1[b]). Our next attempt involved fitting for frequency and frequency derivative jumps $(\Delta \nu$ and $\Delta \dot{\nu})$ at each periastron epoch, similar to 


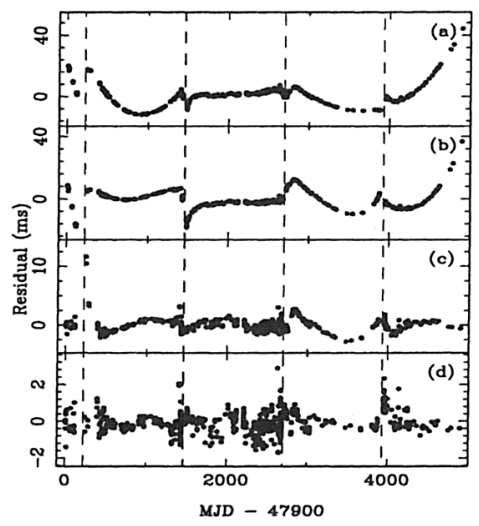

Figure 1. Timing residuals for PSR B1259-63 over 5000 days. (a) fitting for $\nu, \dot{\nu}, \ddot{\nu}$ and the five Keplerian orbital parameters. Addition of: (b) $\dot{\omega}, \dot{x}$; (c) jumps in $\nu$ and $\dot{\nu}$ at each periastron; (d) jumps in $x$ at each periastron.

fits by Manchester et al. (1995) on an early data set. The fit is much improved but some systematic variations are still seen (Fig. 1[c]).

The next attempt was to test the effect of steps in the orbital parameters occurring at periastron. A new binary model (BTJ) based on the BT model (Blandford \& Teukolsky 1976) was implemented in TEMPO to allow cumulative steps in Keplerian orbital parameters. Fitting for the 1997 period glitch in addition to steps in the projected semi-major axis at each periastron produced the almost featureless residuals shown in Figure 1(d).

The effect of timing noise is difficult to quantify because of coupling with the orbital parameters and the long-duration eclipses at each periastron. However the model containing the MJD 50691 glitch and steps in projected semi-major axis at each periastron provides an excellent fit to the 13 years of data. If real, these steps must result from step changes in the orbit inclination.

A paper is in preparation detailing these results and discussing the physical implications for the evolution of the binary system.

Acknowledgments. The Parkes radio telescope is part of the Australia Telescope which is funded by the Commonwealth of Australia for operation as a National Facility by CSIRO.

\section{References}

Blandford R., \& Teukolsky S. A., 1976, ApJ, 205, 580

Johnston S. et al. 1992a, MNRAS, 255, 401

- 1992b, ApJ, 387, L37

- 1996, MNRAS, 279, 1026

- 2001, MNRAS, 326, 643

Manchester R. N. et al. 1995, ApJ, 445, L137

Wex N. et al. 1998, MNRAS, 298, 997 